\title{
The Landmark Series: Systemic Therapy for Resectable Gastrointestinal Stromal Tumors
}

\author{
Emily Z. Keung, $\mathrm{MD}^{1}$, Chandrajit P. Raut, $\mathrm{MD}^{2}$, and Piotr Rutkowski, MD, $\mathrm{PhD}^{3}$ \\ ${ }^{1}$ The University of Texas MD Anderson Cancer Center, Houston, TX; ${ }^{2}$ Brigham and Women's Hospital/Dana-Farber \\ Cancer Institute, Harvard Medical School, Boston, MA; ${ }^{3}$ Maria Sklodowska-Curie National Research Institute of \\ Oncology, Warsaw, Poland
}

\begin{abstract}
Gastrointestinal stromal tumors (GISTs) are the most common mesenchymal neoplasms of the gastrointestinal tract. Complete resection is the only potentially curative treatment, although recurrence is common, occurring in approximately $40-50 \%$ of patients. The introduction of effective molecularly targeted therapies for GISTs has dramatically changed the clinical management paradigms for, and prognosis of, patients with intermediate- and high-risk GISTs, as well as those with locally advanced and metastatic disease. In this article, we review landmark studies that evaluated the use and efficacy of the tyrosine kinase inhibitors imatinib and sunitinib in the adjuvant and neoadjuvant settings for resectable primary and limited resectable metastatic GISTs.
\end{abstract}

Gastrointestinal stromal tumors (GISTs) are the most common mesenchymal tumors of the gastrointestinal tract, developing most commonly in the stomach and small intestine as a result of activating mutations in KIT or PDGFRA, genes encoding receptor protein tyrosine kinases. Over the past 2 decades, remarkable advances have been made in our understanding of GISTs and the development of molecular-targeted therapies, and tyrosine kinase inhibitors (TKIs) such as imatinib and sunitinib have dramatically changed the management and prognosis of patients with this malignancy. Although surgery is the treatment of choice and the only curative treatment for

(C) The Author(s) 2020

First Received: 26 April 2020

Accepted: 4 July 2020;

Published Online: 30 July 2020

P. Rutkowski, MD, PhD

e-mail: piotr.rutkowski@pib-nio.pl resectable GISTs, recurrence is common, particularly in patients with intermediate- and high-risk GISTs as defined by Miettinen and Lasota. ${ }^{1}$ On the heels of landmark clinical trials demonstrating remarkable response of imatinib and sunitinib in patients with advanced unresectable and metastatic GIST, ${ }^{2-4}$ there arose great interest in evaluating the safety and efficacy of using TKI therapy in the adjuvant and neoadjuvant settings for patients with resectable intermediate- and high-risk, locally advanced, or limited resectable metastatic disease. The landmark studies evaluating the use of imatinib for perioperative therapy in resectable GISTs are reviewed below.

\section{ADJUVANT THERAPY}

The success of imatinib in the treatment of advanced unresectable and metastatic GIST ${ }^{2-4}$ led to great interest in using the drug in the adjuvant setting following primary tumor resection to prevent or delay recurrence and prolong survival. The role of imatinib in the adjuvant setting has been evaluated in several phase II and III clinical trials, as summarized below (Table 1).

\section{ACOSOG Z9000}

Purpose and Study Design

The American College of Surgeons Oncology Group (ACOSOG) performed the first trials of imatinib in the adjuvant setting. ACOSOG $\mathrm{Z} 9000^{5}$ was a multicenter, single-arm, phase II study that enrolled 106 patients between September 2001 and September 2003, from 48 institutions, who underwent macroscopically complete resection of high-risk KIT-positive GISTs, defined as tumors $\geq 10 \mathrm{~cm}$, those with intraperitoneal tumor rupture, 


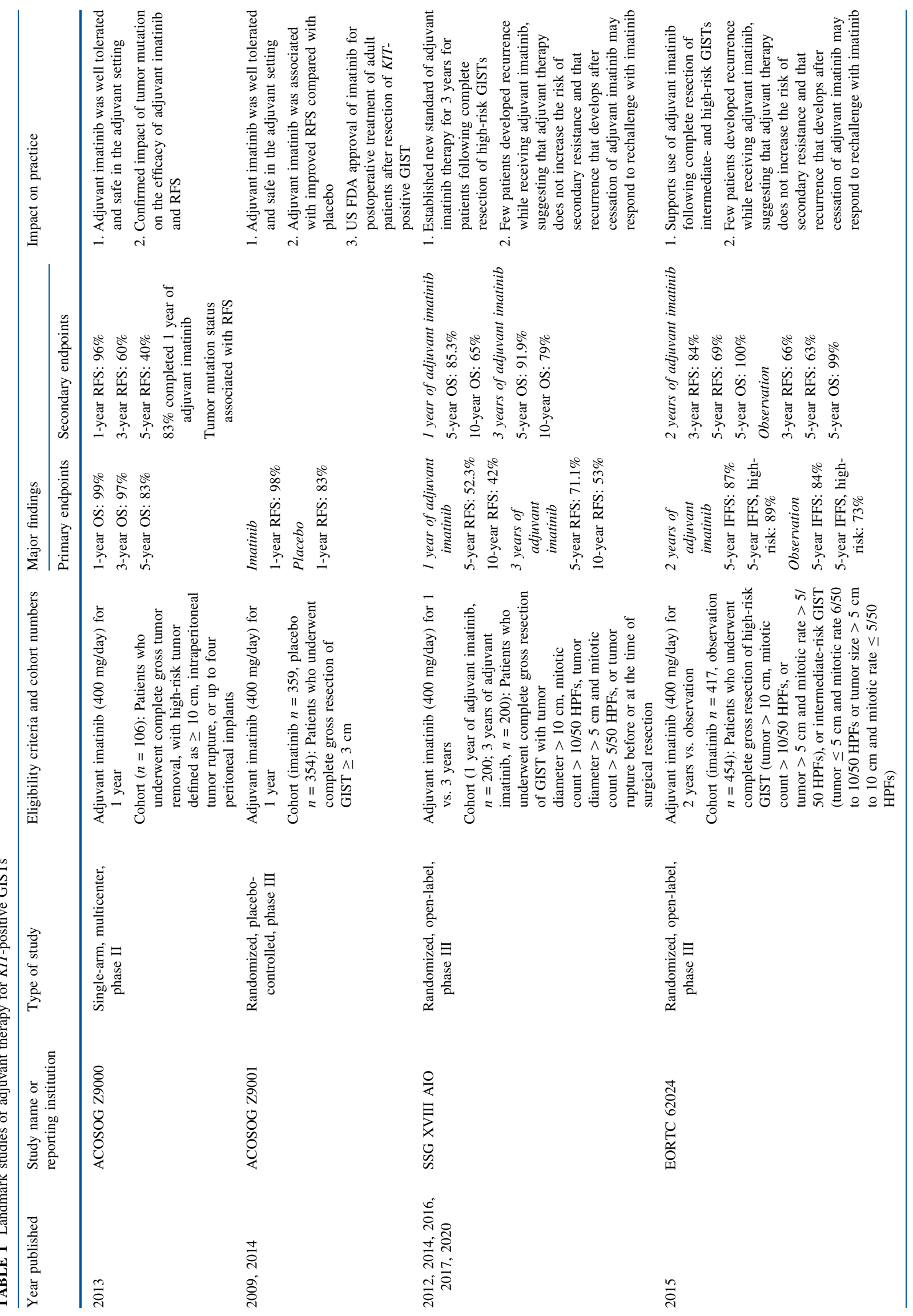




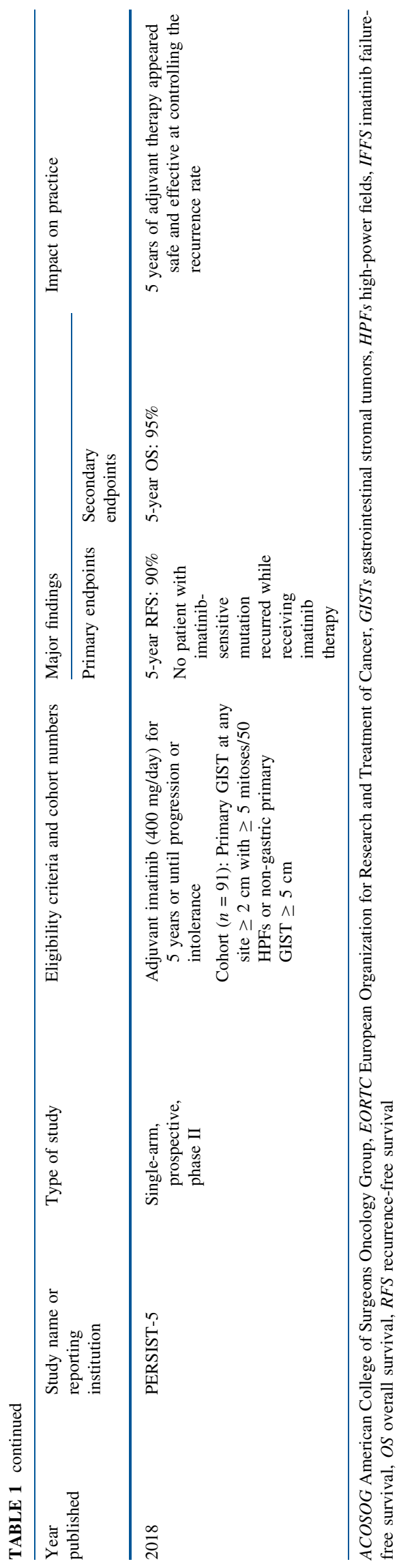

or those with up to four peritoneal implants. Patients received adjuvant imatinib (400 $\mathrm{mg} /$ day) for 1 year and were imaged by computed tomography (CT) or magnetic resonance imaging every 5 months for the first 2 years then every 6 months for the following 3 years. The primary endpoint was to compare overall survival (OS) with that of historical controls, which was estimated to be $35 \%$ with surgery alone.

\section{Results}

Overall, in this single-arm study, adjuvant imatinib was well tolerated and safe, with $83 \%$ of patients completing the prescribed year of therapy and $69 \%$ receiving the prescribed dose of $400 \mathrm{mg} /$ day. The results of this study showed that postoperative imatinib for 1 year prolonged recurrence-free survival (RFS) after complete GIST resection and was also associated with improved OS compared with historic controls (3-year OS 97\%, 5-year OS $83 \%$ vs. historic 5-year OS 35\%). As expected, tumor mitotic rate, tumor location, and mutation status were associated with RFS on univariate analyses.

\section{Implications for Practice}

ACOSOG Z9000/Z9001 showed that adjuvant imatinib for 1 year following macroscopically complete resection of primary GISTs was safe and well tolerated and improved RFS compared with placebo. Based on the results of ACOSOG Z9001, in December 2008 the US FDA approved imatinib for the postoperative treatment of adult patients after resection of KIT-positive GISTs. However, as many patients in both Z9000 and Z9001 recurred upon completion of therapy, the results of these studies suggested that adjuvant imatinib delays rather than prevents disease recurrence and that longer duration of adjuvant therapy may further improve RFS.

\section{ACOSOG Z9001}

\section{Purpose and Study Design}

ACOSOG Z9001 $1^{6,7}$ was a randomized, double-blind, placebo-controlled, phase III trial of adjuvant imatinib for 1 year in patients who underwent complete gross resection of primary KIT-positive GISTs $\geq 3 \mathrm{~cm}$ (Fig. 1). Patients from 230 institutions were randomized to receive either adjuvant imatinib $(n=359)$ or placebo $(n=354)$; crossover was allowed in the event of tumor recurrence. Although the original primary endpoint was OS, 6 months prior to the first planned efficacy interim analysis the primary endpoint was changed to RFS when it became clear 
FIG. 1 ACOSOG Z9001 study schema. ACOSOG American College of Surgeons Oncology Group, GIST gastrointestinal stromal tumor, ECOG Eastern Cooperative Oncology Group, $C T$ computed tomography, $M R I$ magnetic resonance imaging
Eligibility Criteria Included:

- Complete gross resection (R0/R1) of $\geq 3$-cm primary GIST

- KIT positive by immunohistochemistry

- At least 18 years of age

- ECOG PS $\leq 2$

- Registered within 70 days after surgery and start therapy by 84 days after surgery

- No evidence of disease on imaging within 28 days of initiation of therapy

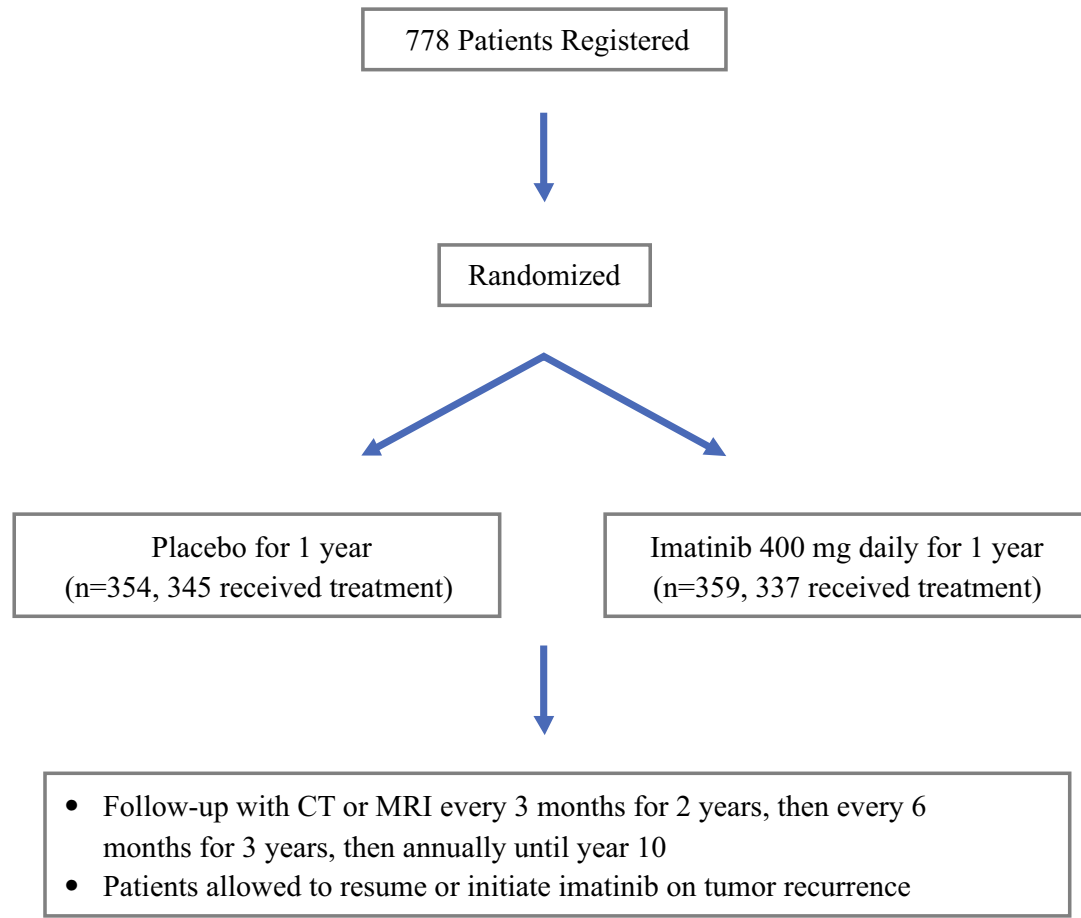

that the event (death) rate would be significantly lower than the event rate specified in the original statistical design because of the efficacy of imatinib and the crossover trial design that allowed patients who progressed on placebo to receive imatinib.

\section{Results}

Median follow-up was 19.7 months (0-56.4), during which $30(8 \%)$ patients in the imatinib group and $70(20 \%)$ in the placebo group developed tumor recurrence or died. Adjuvant imatinib was associated with significantly improved RFS compared with placebo $(98 \%$ vs. $83 \%)$ at 1 year and was well tolerated. No difference in OS was noted; however, this was not unexpected given the crossover design. Of note, patients who recurred generally did so shortly after completion of 1 year of adjuvant therapy. The slope of the RFS curve for the patients in the imatinib arm was similar to that of the placebo arm, but offset by approximately 18 months. Additionally, Z9001 enrolled many patients considered to be at low risk of recurrence, as subsequently defined by Miettinen and Lasota, as the risk categories were not well understood at the start of the trial. ${ }^{1}$

\section{SCANDINAVIAN/GERMAN SSG XVIII/AIO}

Purpose and Study Design

The Scandinavian/German SSG XVIII/AIO trial ${ }^{8-12}$ was a randomized, open-label trial of 1 versus 3 years of postoperative imatinib (400 mg/day) after complete gross resection of high-risk KIT-positive GISTs following complete macroscopic resection (Fig. 2). High-risk GIST was defined as tumors with at least one of the following: tumor diameter $>10 \mathrm{~cm}$, mitotic count $>10 / 50$ high-power fields (HPF), tumor diameter $>5 \mathrm{~cm}$ and mitotic count $>5 / 50$ HPFs, or tumor rupture before or at the time of surgical resection. A total of 400 patients from 24 hospitals were enrolled between 4 February 2004 and 29 September 2008. 
FIG. 2 SSG XVIII/AIO study schema. GIST gastrointestinal stromal tumor, ECOG PS

Eastern Cooperative Oncology Group performance score, $C T$ computed tomography, MRI magnetic resonance imaging
Eligibility Criteria Included:

- Complete gross resection (R0/R1) of GIST at high estimated risk of recurrence defined as one of the following

- >10-cm GIST, or

- $>10$ mitoses per 50 high-power field, or

- $>5$-cm GIST and > 5 mitoses per 50 high-power field, or

- Tumor rupture at time of surgery

- KIT positive by immunohistochemistry

- At least 18 years of age

- ECOG PS $\leq 2$

- Randomized within 12 weeks of surgery

- No evidence of disease on imaging within 28 days of initiation of therapy
Imatinib $400 \mathrm{mg}$ daily for 12 months $(\mathrm{n}=200)$

\section{Patients Registered}

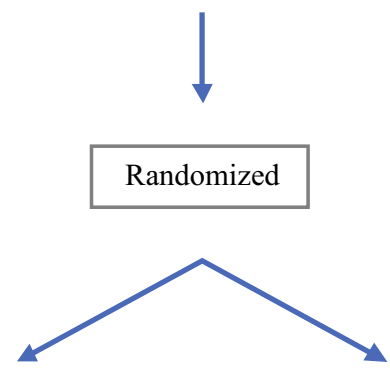

Imatinib $400 \mathrm{mg}$ daily for 36 months $(n=200)$

- Follow-up with CT or MRI every 6 months during treatment and follow-up until month 84 of study, then annually

\section{Results}

Results after 10 years of follow-up were recently presented. ${ }^{12}$ Median follow-up was 119 months. Compared with patients randomized to receive 1 year of adjuvant imatinib, those randomized to receive 3 years of adjuvant imatinib were observed to have improved 5-year/10-year RFS (71/53\% vs. 53/42\%; hazard ratio [HR] 0.66, 95\% confidence interval [CI] $0.49-0.87 ; p=0.003$ ) and 5-year/ 10-year OS $(92 / 79 \%$ vs. $86 / 65 \%$; HR 0.55 , $95 \%$ CI $0.37-0.83 ; p=0.04)$.

\section{Implications for Practice}

This study established a new standard for treating patients after resection of high-risk GISTs with adjuvant imatinib for 3 years. The long-term data confirmed sustained OS benefit in the 3-year adjuvant imatinib arm. Of note, this study did include patients with GISTs now understood to be less sensitive to imatinib at $400 \mathrm{mg} /$ day dosing (KIT exon 9 mutation), as well as those likely to be primarily resistant to imatinib (wild-type, PDGFRA D842V), with similar numbers in each trial arm.

\section{EORTC $62024^{13}$}

\section{Purpose and Study Design}

The European Organization for Research and Treatment of Cancer (EORTC) conducted the randomized, open-label, phase III EORTC 62024 trial $^{13}$ to compare 2-year adjuvant treatment with imatinib versus observation alone in patients with intermediate- and high-risk primary KITpositive GISTs (Fig. 3). The primary endpoint of this study 
FIG. 3 EORTC 62024 study schema. EORTC European Organization for Research and Treatment of Cancer, GIST gastrointestinal stromal tumor, ECOG PS Eastern Cooperative Oncology Group performance score, $C T$ computed tomography, MRI magnetic resonance imaging
Eligibility Criteria Included:

- Complete gross resection (R0/R1, with tumor rupture coded as R1) of GIST at intermediate or high estimated risk of recurrence defined as one of the following

- >10-cm GIST, or

- $>10$ mitoses per 50 high-power field, or

- $>5$-cm GIST and $>5$ mitoses per 50 high-power field, or

- $\leq 5$-cm GIST and 6-10 mitoses per 50 high-power field, or

- $>5$ to $10 \mathrm{~cm}$ GIST and > 5 mitoses per 50 high-power field

- KIT positive by immunohistochemistry

- At least 18 years of age

- ECOG PS $\leq 2$

- Randomized between 2 weeks to 3 months of surgery

- No evidence of disease on imaging within 28 days of initiation of therapy

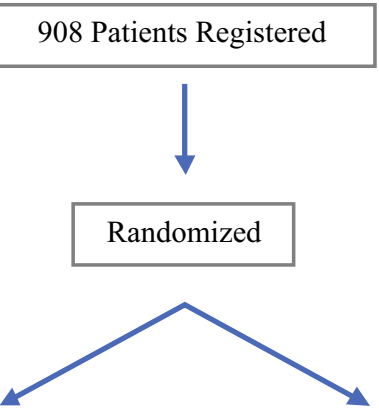

Imatinib $400 \mathrm{mg}$ daily for 2 years $(\mathrm{n}=454)$

- Follow-up with CT or MRI every 3 months for 2 years, then every 4 months for 3 years, then at least annually

- Patients allowed to resume or initiate imatinib or start a tyrosine kinase inhibitor other than imatinib on tumor relapse

was originally OS but was modified in 2009 to imatinib failure-free survival (IFFS), defined as time to death or starting a TKI other than imatinib, as an endpoint sensitive to secondary resistance to imatinib. Of 908 patients who were enrolled from 112 hospitals between December 2004 and October 2008, 835 patients were eligible, with 418 randomized to the observation arm and 417 randomized to the adjuvant imatinib arm.

\section{Results}

Median follow-up was 56.4 months. There was no significant difference in IFFS between study arms, with 402 patients remaining imatinib failure-free in the imatinib arm and 391 patients remaining imatinib failure-free in the observation arm (3-year IFFS $90.8 \%$ vs. 90.8\%, 5-year
IFFS 87.0\% vs. 84.1\%; HR 0.79 , 98.5\% CI 0.5-1.25; $p=0.21)$. RFS was significantly better among patients who received adjuvant imatinib (3-year RFS $84.3 \%$ vs. $65.8 \%$, 5 -year RFS $69.4 \%$ vs. $62.9 \%$; $p<0.01$ ). OS did not differ between arms (3-year OS $96.8 \%$ vs. 96.3\%, 5-year OS $91.8 \%$ vs. $92.7 \%$ ).

\section{Implications for Practice}

This study again supported the role of adjuvant imatinib in decreasing recurrence risk for patients with intermediate- and high-risk GISTs following complete macroscopic resection. However, the broader implication is that there is no difference in time to the start of second-line therapy whether imatinib is administered as an adjuvant therapy immediately after surgery or after recurrence. 


\section{PERSIST-5 $^{14}$}

\section{Purpose and Study Design}

As 3 years of adjuvant imatinib therapy was shown to be associated with reduced recurrence rates and improved OS in patients with high-risk primary GISTs compared with those who received 1 year of therapy, the question arose as to whether a longer duration of adjuvant imatinib beyond 3 years might improve outcomes further. PERSIST-5 is a prospective, multicenter, single-arm, phase II study to evaluate whether adjuvant treatment with imatinib $(400 \mathrm{mg} /$ day) for 5 years is tolerable and efficacious. Eligible patients included those with a primary GIST of any location $\geq 2 \mathrm{~cm}$ with $\geq 5$ mitoses $/ 50 \mathrm{HPFs}$, or primary non-gastric GIST $\geq 5 \mathrm{~cm}$. Ninety-one patients from 21 institutions were enrolled, with data collected from 5 August 2009 through 20 December 2016. Primary and secondary endpoints included 5-year RFS and OS, respectively.

\section{Results}

Twenty-four (26\%) patients had GISTs that were of intermediate risk of recurrence based on the Miettinen and Lasota classification, ${ }^{1}$ while $67(74 \%)$ were of high risk. Median duration of adjuvant imatinib treatment was 55.1 months (range 0.5-60.6), with 46 patients $(51 \%$ ) completing 5 years of therapy. Forty-five patients (49\%) discontinued treatment before 5 years because of patient choice $(21 \%)$, adverse events $(16 \%)$, or other reasons (12\%). No patient with imatinib-sensitive mutations had disease recurrence while receiving therapy. Of the seven patients who recurred, one recurred while receiving imatinib but had PDGFRA D842V mutation, and six recurred after discontinuation of imatinib therapy.

\section{Implications for Practice}

Adjuvant therapy with imatinib for 5 years was demonstrated to be safe and effective at controlling recurrence rates in patients with imatinib-sensitive mutations. However, compliance with this adjuvant regimen was challenging, with $49 \%$ of patients discontinuing therapy early. The results of the study emphasized the importance of pretreatment assessment of GIST molecular status as a driver of long-term benefit with adjuvant targeted therapy. It is unknown whether this longer 5-year duration of adjuvant imatinib therapy is associated with improved RFS and OS compared with 3-year adjuvant therapy, although the results appear comparable with those of EORTC 62024 and SSG XVIII/AIO. The results of this single-arm exploratory study is the basis of a randomized trial of 3-year versus 5-year adjuvant imatinib therapy (NCT 02413736).

\section{NEOADJUVANT TREATMENT}

The remarkable responses to imatinib in many patients with advanced unresectable and metastatic GISTs prompted the question of whether a neoadjuvant imatinib treatment approach might benefit patients in select clinical circumstances. Neoadjuvant therapy is an attractive treatment strategy to downstage disease, allow definitive resection, and improve local disease control in patients with locally advanced and/or marginally resectable solid tumors across histologies for whom upfront surgery may be technically challenging, overly morbid, or not feasible. Current European (European Society for Medical Oncology [ESMO]) and US (National Comprehensive Cancer Network $[\mathrm{NCCN}]$ ) guidelines ${ }^{15,16}$ recommend consideration of preoperative imatinib and tumor mutational testing if surgical morbidity could be reduced by downstaging the tumor preoperatively. This approach is particularly attractive and indicated in cases when surgery may be technically challenging (rectum, duodenum, gastroesophageal junction) and tumor downstaging may facilitate tumor resectability (converting from an open laparotomy to a minimally invasive approach) or enable a less extensive/organ-sparing surgery, and may improve surgical outcomes such as likelihood of achieving a negative margin of resection and decreasing the risk of tumor perforation/rupture. Preoperative therapy with imatinib should be used until the maximum response is obtained (usually 6-12 months from the beginning of treatment) and before the development of secondary resistance to therapy. Response to therapy should be carefully monitored by imaging studies. As accurate assessment of recurrence risk cannot be made in patients who received preoperative systemic therapy, adjuvant imatinib should be used for at least 3 years following surgical resection. These recommendations are based on a limited number of landmark phase II and collaborative retrospective studies described below (Table 2).

\section{RTOG 0132/ACRIN $6665^{17,18}$}

\section{Purpose and Study Design}

The Radiation Therapy Oncology Group (RTOG) 01321 was a prospective, multicenter, non-randomized, phase II trial evaluating the efficacy and tolerability of preoperative imatinib in patients with $K I T$-positive resectable intermediate- to high-risk primary $(\geq 5 \mathrm{~cm})$ or recurrent/ metastatic $(\geq 2 \mathrm{~cm})$ GISTs. Patients were enrolled from 18 RTOG institutions and were treated with imatinib 


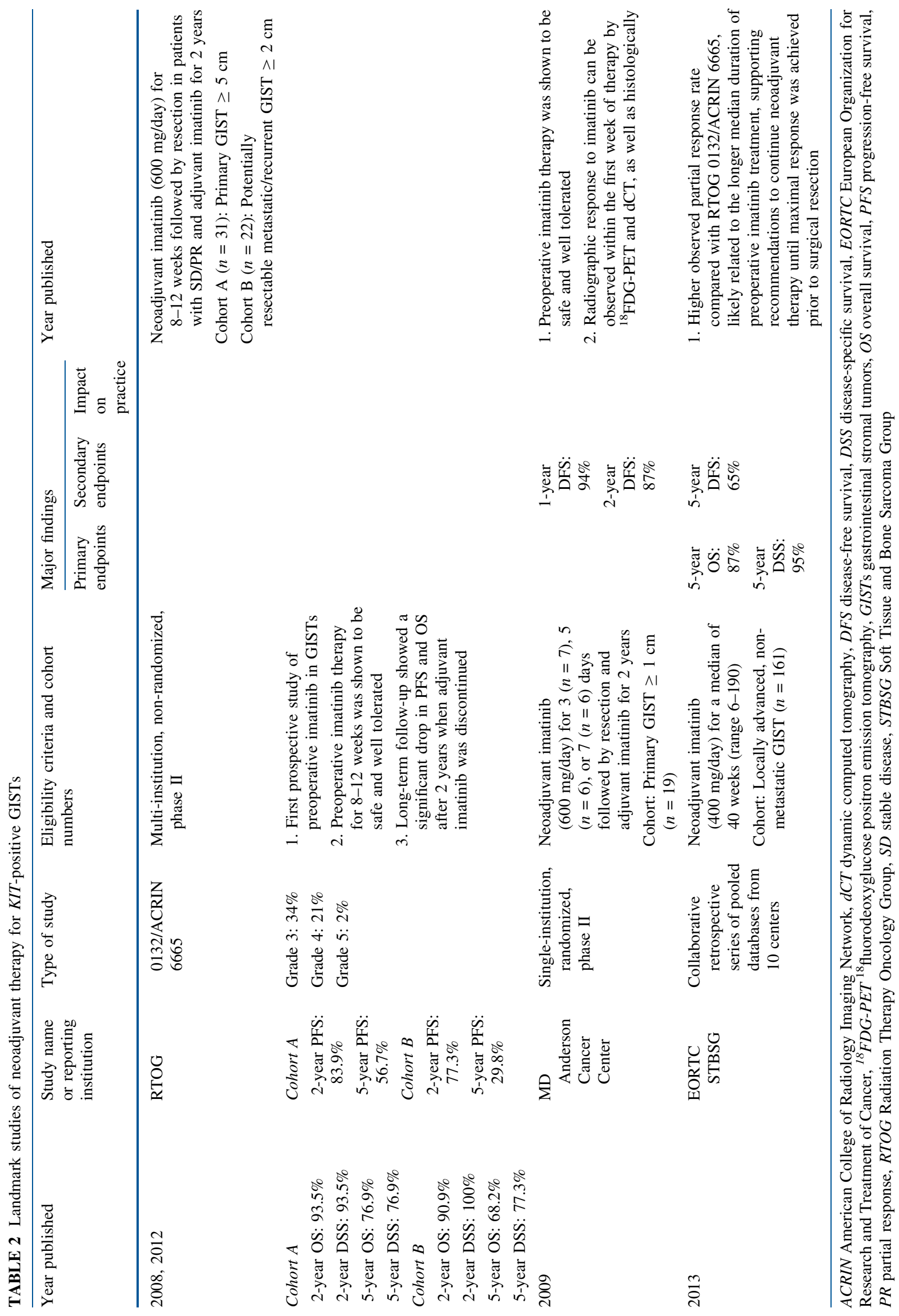


(600 mg/day) for $8-12$ weeks prior to surgery. Imatinib was stopped on the day prior to surgery and resumed as soon as possible postoperatively for 2 years. Clinical endpoints included assessments of imatinib-related toxicity, surgical complication assessment, GIST response to preoperative therapy, time to progression, progression-free survival (PFS), disease-specific survival, and OS.

\section{Results}

Most primary GISTS $(n=30)$ presented in the stomach $(52 \%)$, followed by the small bowel (20\%), and median tumor size was $8.7 \mathrm{~cm}$ (range 5-24.5). Among patients with primary GISTs, stable disease (SD) and partial response (PR) were observed in $83 \%$ and $7 \%$ of patients, respectively, while in those with recurrent or metastatic disease $(n=22)$, SD and PR were observed in $91 \%$ and $4.5 \%$ of patients, respectively. Estimated 2-year OS and PFS were $93.5 \%$ and $83.9 \%$, respectively, for patients with primary GIST, and $90.9 \%$ and $77.3 \%$, respectively, for patients with recurrent or metastatic disease. Overall, neoadjuvant imatinib was well tolerated, with rates of grade 3,4 , and 5 postoperative toxicities of $34 \%, 20.8 \%$, and $1.9 \%$, respectively. The 8 - to 12 -week delay in the time to surgery did not appear to have any adverse effects on surgical outcomes.

\section{Implications for Practice}

Initial results of RTOG 0132/American College of Radiology Imaging Network (ACRIN) 6665 were reported in 2008 and demonstrated that preoperative imatinib for 8-12 weeks was safe and well tolerated. Although survival outcomes at 2 years compared favorably with historical single-institution surgical series for patients with intermediate- to high-risk GISTs, it was unclear whether the apparent survival benefits seen in this study could be attributed to the use of imatinib preoperatively, or due to the 2 years of postoperative imatinib therapy. Follow-up results of this study were reported in 2012 and were notable for a significant drop in PFS and OS after 2 years when adjuvant imatinib was discontinued, supporting the need for longer durations of imatinib treatment in patients at intermediateand high-risk of GIST recurrence following resection.

\section{ANDERSON, MCAULIFFE ET AL. ${ }^{19}$}

\section{Purpose and Study Design}

The group at The University of Texas MD Anderson Cancer Center (McAuliffe et al. ${ }^{19}$ ) performed a prospective, single-institution study to evaluate the safety and efficacy of preoperative imatinib (600 mg/day) in 19 patients with $K I T$-positive resectable GISTs $(\geq 1 \mathrm{~cm})$. Patients were randomized to receive imatinib for 3,5 , or 7 days preoperatively ( $n=7,6$, and 6 , respectively), with the last dose administered the morning of surgery. Preoperative tumor response to imatinib was assessed radiographically by dynamic CT (dCT) and ${ }^{18}$ fluorodeoxyglucose positron emission tomography $\left({ }^{18} \mathrm{FDG}-\right.$ PET), as well as histologically. Imatinib was resumed postoperatively for 2 years.

\section{Results}

This study provided evidence that radiographic response to preoperative imatinib can be observed and assessed as early as within 1 week of initiation of therapy. Most patients in this study responded to preoperative imatinib as assessed by ${ }^{18}$ FDG-PET and dCT $(69 \%$ and $71 \%$, respectively). Similar to the results of RTOG 0132/ACRIN 6665, preoperative imatinib was well tolerated and safe. The observed 2-year disease-free survival (DFS) was $87 \%$, with a median DFS of 46 months. Survival benefit of preoperative imatinib could not be determined as all patients received imatinib postoperatively for 2 years.

\section{Implications for Practice}

Although a small study $(n=19)$, this single-institution, phase II trial demonstrated that preoperative imatinib was safe, and additionally showed that tumor metabolic response to TKI therapy occurs and can also be assessed within 1 week of treatment by ${ }^{18}$ FDG-PET.

\section{EORTC STBSG ${ }^{20}$}

\section{Purpose and Study Design}

The EORTC Soft Tissue and Bone Sarcoma Group (STBSG) performed a retrospective study to assess the long-term results of preoperative imatinib therapy in 161 consecutive patients with primary KIT-positive GISTs treated with preoperative imatinib between 2002 and 2011 at 10 sarcoma centers of the EORTC-STBSG. This remains the largest study to date of neoadjuvant imatinib therapy. Preoperative imatinib was continued until either maximal response, defined as two consecutive CT scans not showing further tumor regression, or if the surgeon deemed that resection was possible, whichever was achieved first.

\section{Results}

Median follow-up was 46 months. Tumors were located in the stomach $(55.3 \%)$, rectum $(20.5 \%)$, duodenum 
(9.9\%), ileum/jejunum (9.3\%), esophagus $(3.1 \%)$, and other locations $(1.9 \%)$. The median time of preoperative imatinib therapy in this study was 40 weeks (range 6-190); 129 patients $(80.1 \%)$ had documented response to preoperative therapy and 30 patients $(18.6 \%)$ had documented stabilization of disease prior to surgery. The median duration of adjuvant imatinib therapy was 19 months (range 12-76). This study reported excellent safety data and long-term results, with a 5-year DFS and OS of $65 \%$ and $87 \%$, respectively.

\section{Implications for Practice}

The number of PRs was higher in this large retrospective series compared with that observed in the RTOG 0132-ACRIN 6665 trial, likely related to the longer duration of preoperative imatinib therapy and thus supporting the recommendation that preoperative imatinib therapy be continued until maximal tumor response is achieved. Survival benefit of preoperative imatinib could not be determined as all patients received imatinib postoperatively.

\section{COMBINATION OF TARGETED THERAPY AND METASTASECTOMY}

Cytoreductive surgery for recurrent or metastatic GISTs may be considered in select circumstances, including in patients presenting with oncologic emergencies such as hemorrhage, intestinal perforation, or obstruction, as well as in patients whose disease is stable or responsive to TKI therapy and when complete gross resection is possible and in patients with limited disease progression. ${ }^{16}$

In the pre-imatinib era, surgical resection of recurrent and metastatic GISTs was associated with improved survival if complete gross resection could be achieved..$^{21}$ However, complete resection was often difficult to achieve due to the multifocal nature of recurrent and metastatic GISTs. In the post-imatinib era, TKI therapy is the standard first-line treatment for patients with metastatic, recurrent, and/or inoperable GISTs. Although maximal response to imatinib is typically achieved within 6-18 months of treatment, ${ }^{22}$ complete remissions are rare and median time to recurrence/ progression on imatinib is within 2 years. ${ }^{23,24}$ Thus, cytoreductive surgery to resect residual disease became an attractive approach to investigate. Although prospective randomized trials did not prove feasible, a number of retrospective series of patients with recurrent and/or metastatic GISTs treated surgically after treatment with imatinib and/or sunitinib therapy have been published supporting the role of surgery for residual metastatic disease in patients with GISTs responding to imatinib (Table 3). ${ }^{24-42}$

\section{CHICTR-TRC-00000244 ${ }^{34}$}

\section{Purpose and Study Design}

Two clinical trials (NCT00956072 in Europe, ChiCTRTRC-00000244 in China) were attempted to address the question of whether patients with metastatic GISTs receiving imatinib would benefit further from surgical resection of residual disease. Both studies were closed early due to poor accrual, although ChiCTR-TRC00000244 reported data on 41 of 210 planned patients. In ChiCTR-TRC-00000244, patients were treated with imatinib $(400 \mathrm{mg} / \mathrm{day})$ and those responding to therapy were randomized to either surgery for resection of residual disease with resumption of imatinib postoperatively $(n=19)$ or imatinib therapy alone $(n=22)$. All patients continued imatinib until disease progression

\section{Results}

Patients in the surgery arm underwent surgery 3-12 months after initiating imatinib therapy, with 15 patients achieving PR and 7 patients achieving SD at the time of randomization. Of those in the imatinib-alone arm, 9 achieved PR and 10 achieved SD at the time of randomization. Two-year PFS was $88.4 \%$ in the surgery arm versus $57.7 \%$ in the imatinib-alone arm, with a median follow-up of 23 months $(p=0.089)$. Median OS was not reached in the surgery arm, and was 49 months in the imatinib-alone arm $(p=0.024)$.

\section{Implications for Practice}

Although there was some suggestion of improved PFS with surgery compared with imatinib therapy alone, the difference was not statistically significant. It is possible that surgery may prolong survival in carefully selected patients, although this decision should be made at expert centers and following multidisciplinary discussion.

\section{RETROSPECTIVE STUDIES EXAMINING DISEASE CONTROL AFTER RESECTION IN SELECTED PATIENTS WITH LIMITED METASTATIC DISEASE AFTER IMATINIB TREATMENT}

Despite the lack of prospective, randomized controlled trials, the conclusions of multiple available retrospective series have overall been consistent. Although complete excision of residual metastatic lesions is associated with improved prognosis in these retrospective series, ${ }^{24-40}$ outcomes are consistently dependent on preoperative responses to imatinib, and it has not been prospectively 
TABLE 3 Landmark studies of metastasectomy for GISTs treated with tyrosine kinase inhibitor therapy

Authors, Number of cases, clinical indications
year
published

\begin{tabular}{|c|c|}
\hline \multirow[t]{4}{*}{ Raut et al. ${ }^{25}$} & $\begin{array}{l}N=69 \text { patients who underwent surgery for advanced } \\
\text { GISTs while receiving TKI therapy }\end{array}$ \\
\hline & Group I $(n=23)$ : surgery at stable disease \\
\hline & Group II $(n=32)$ : surgery at limited progression \\
\hline & Group III $(n=14)$ : surgery at generalized progression \\
\hline \multirow[t]{3}{*}{$\begin{array}{l}\text { Rutkowski } \\
\text { et al. }{ }^{42}\end{array}$} & $\begin{array}{l}N=141 \text { patients who underwent surgery for initially } \\
\text { inoperable and/or metastatic GISTs after receiving } \\
\text { imatinib therapy }\end{array}$ \\
\hline & $\begin{array}{l}\text { Group I }(n=24) \text { : resection of residual disease after } \\
\text { complete/partial response or lack of further response }\end{array}$ \\
\hline & $\begin{array}{l}\text { Group II }(n=8) \text { : surgery as salvage therapy for } \\
\text { progression after initially successful imatinib therapy }\end{array}$ \\
\hline \multirow[t]{3}{*}{$\begin{array}{l}\text { Gronchi } \\
\text { et al. }{ }^{39}\end{array}$} & $\begin{array}{l}N=159 \text { patients with advanced/metastatic GISTs treated } \\
\text { initially with imatinib }\end{array}$ \\
\hline & Group I $(n=27)$ : surgery at response \\
\hline & Group II $(n=8)$ : surgery at progression \\
\hline \multirow[t]{4}{*}{$\begin{array}{l}\text { DeMatteo } \\
\text { et al. }\end{array}$} & $\begin{array}{l}N=40 \text { patients with metastatic GISTs treated with TKI } \\
\text { therapy }\end{array}$ \\
\hline & Group I $(n=20)$ : surgery at response \\
\hline & Group II $(n=13)$ : surgery at focal progression \\
\hline & Group III $(n=7)$ : surgery at multifocal progression \\
\hline \multirow[t]{3}{*}{$\begin{array}{l}\text { Mussi } \\
\text { et al. }\end{array}$} & $\begin{array}{l}N=80 \text { patients with metastatic GISTs treated with TKI } \\
\text { therapy }\end{array}$ \\
\hline & Group I $(n=49)$ : surgery at best response \\
\hline & Group II $(n=31)$ : surgery at focal progression \\
\hline
\end{tabular}

Raut et al. ${ }^{36} \quad N=50$ patients with metastatic imatinib-resistant GISTs undergoing surgery following sunitinib therapy

Group I $(n=10)$ : responsive disease

Group II $(n=22)$ : limited progression

Group III $(n=18)$ : generalized progression
Tielen $\quad N=55$ patients with advanced/metastatic GISTs treated et al. ${ }^{35} \quad$ with TKI therapy
Group I $(n=35)$ : responders
Group II $(n=20)$ : nonresponders
Bauer et $\mathrm{al}^{26}$
$N=239$ patients with GISTs undergoing surgery for metastatic GISTs

Group I ( $n=177)$ : complete gross resection (R0/R1)

Group II $(n=62)$ : incomplete gross resection (R2)
Du et al. ${ }^{34} \quad N=41$ of 210 planned patients with recurrent/metastatic GISTs treated with TKI therapy
Arm A $(n=19)$ : surgery
Arm B $(n=22)$ : imatinib alone
Fairweather 400 operations performed in 323 patients with metastatic et al. ${ }^{33}$ GISTs treated with TKI therapy
Group $1(n=64)$ : surgery at response

Group I: 1-year PFS 80\%, 1-year OS 95\%

Group II: 1-year PFS 33\%, 1-year OS 86\%

Group III: 1-year PFS 0\%, 1-year OS 0\%

Median follow-up 12 months

Group I: Four recurrences of 5 patients who did not resume imatinib postoperatively, 1 recurrence of 19 patients who resumed imatinib postoperatively

Group II: $5 / 8$ patients progressed

Group I: 1-year PFS 96\%, 1-year OS 100\%; 2-year PFS 69\%

Group II: 1-year PFS 0\%, 1-year OS 60\%

Median follow-up 15 months

Group I: 2-year PFS 61\%, 2-year OS 100\%

Group II: 2-year PFS 24\%, 2-year OS 36\%, median TTP 12 months

Group III: 1-year OS 36\%, median TTP 3 months

Morbidity in 13 patients

Group I: 2-year PFS 64.4\%, median PFS not reached, 5-year DSS $82.9 \%$, median DSS not reached

Group II: 2-year PFS 9.7\%, median PFS 8 months, 5-year DSS $67.6 \%$, median DSS not reached

Complication rate $54 \%, 48 \% \mathrm{R} 2$ resection

Median follow-up 15.2 months

Median PFS and OS after surgery 5.8 and 16.4 months, respectively

Median PFS and OS after the start of sunitinib 15.6 and 26 months, respectively

Differences in PFS and OS between groups were not significant

Group I: $48 \%$ recurrence/progression, median PFS and OS not reached, 5-year OS 78\%

Group II: $85 \%$ recurrence/progression, median PFS 4 months, median OS 25 months, 3-year OS $26 \%$

Group I: median OS 8.7 years, median OS was not reached when surgery was performed at remission, median TTP was not reached

Group II: median OS 5.3 years, median OS 5.1 years when surgery was performed at remission, median TTP 1.9 years when surgery was performed at response

Groups I and II: no difference in median PFS in patients progressing at the time of surgery

Group I: 2-year PFS $88.4 \%$, median OS not reached

Group II: 2-year PFS $57.7 \%$, median OS 49 months

In patients receiving imatinib before surgery, radiographic response was predictive of PFS and OS from the time of surgery

Group 1: PFS 36 months, OS not reached 
Table 3 (continued)

\begin{tabular}{lll}
\hline $\begin{array}{l}\text { Authors, } \\
\text { year } \\
\text { published }\end{array}$ & Number of cases, clinical indications & Key results \\
\hline & Group 2 $(n=100)$ : surgery at stable disease & Group 2: PFS 30 months, OS 144 months \\
& Group 3 $(n=132)$ : surgery at limited progression & Group 3: PFS 11 months, OS 105 months \\
& Group 4 $(n=104)$ : surgery at generalized progression & Group 4: PFS 6 months, OS 66 months \\
\hline
\end{tabular}

Adapted from Rutkowski and Hompes ${ }^{22}$

DSS disease-specific survival, GISTs gastrointestinal stromal tumors, OS overall survival, PFS progression-free survival, TKI tyrosine kinase inhibitor, TTP time to progression

demonstrated that the survival benefit associated with surgical resection of residual metastatic disease is due to surgery itself or to patient selection.

Currently available data do not support a clinical benefit of surgery for patients with generalized disease progression on TKI therapy. ${ }^{25,38,42}$ Raut et al. reported that 1-year PFS following surgical resection was $80 \%, 33 \%$ and $0 \%$ in patients with advanced GIST who achieved SD, limited progression, and generalized progression, respectively, with imatinib therapy preoperatively. ${ }^{25}$ Similarly, Rutkowski et al. found that patients who achieved PR or SD had significantly longer PFS and OS following surgical resection when compared with patients with progressive disease. The role of surgery in patients with advanced GISTs with focally progressive disease on imatinib, as well as those on lines of systemic therapy beyond imatinib, is limited $^{23,36}$ and should be individualized and considered in an expert and multidisciplinary setting.

\section{CONCLUSION}

The development of TKIs has dramatically altered the management landscape and improved outcomes of patients with GISTs. Initially limited to use in the metastatic setting, TKIs have since been shown to have utility both in the neoadjuvant and adjuvant settings. The rationale use of TKI therapy in the metastatic, neoadjuvant, and adjuvant settings requires knowledge of GIST mutational status, obtained through tumor biopsies prior to initiation of systemic therapy. Although imatinib and subsequent generations of TKIs have to date primarily benefitted patients with GISTs harboring common KIT mutations, avapritinib, the most recent TKI to receive FDA approval, demonstrates efficacy in patients with GISTs harboring the PGDFR D842V mutation. Future work will likely evaluate novel therapies, including avapritinib in combination with surgical management, for growing subsets of patients with GIST.
DISCLOSURES Piotr Rutkowski has received honoraria for lectures and advisory board work from Novartis, MSD, BMS, Pfizer, Pierre Fabre, Roche, Eli Lilly, Blueprint Medicines.

OPEN ACCESS This article is licensed under a Creative Commons Attribution 4.0 International License, which permits use, sharing, adaptation, distribution and reproduction in any medium or format, as long as you give appropriate credit to the original author(s) and the source, provide a link to the Creative Commons licence, and indicate if changes were made. The images or other third party material in this article are included in the article's Creative Commons licence, unless indicated otherwise in a credit line to the material. If material is not included in the article's Creative Commons licence and your intended use is not permitted by statutory regulation or exceeds the permitted use, you will need to obtain permission directly from the copyright holder. To view a copy of this licence, visit http://creativecommons. org/licenses/by/4.0/.

\section{REFERENCES}

1. Miettinen M, Lasota J. Gastrointestinal stromal tumors: pathology and prognosis at different sites. Semin Diagn Pathol. 2006;23(2):70-83.

2. Demetri GD, von Mehren M, Blanke CD, van den Abbeele AD. Efficacy and safety of Imatinib mesylate in advanced gastrointestinal stromal tumors. N Engl J Med. 2002;347(7):472-80.

3. Blanke CD, Demetri GD, Von Mehren M, et al. Long-term results from a randomized phase II trial of standard-versus higher-dose imatinib mesylate for patients with unresectable or metastatic gastrointestinal stromal tumors expressing KIT. J Clin Oncol. 2008;26(4):620-5.

4. Demetri GD, van Oosterom AT, Garrett CR, et al. Efficacy and safety of sunitinib in patients with advanced gastrointestinal stromal tumour after failure of imatinib: a randomised controlled trial. Lancet. 2006;368(9544):1329-38.

5. Dematteo RP. Long-term results of adjuvant imatinib mesylate in localized, high-risk, primary gastrointestinal stromal tumor. Ann Surg. 2013;258:422-9.

6. Dematteo RP, Ballman K V, Antonescu CR, et al. Adjuvant imatinib mesylate after resection of localised, primary gastrointestinal stromal tumour: a randomised, double-blind, placebocontrolled trial. Lancet. 2009;373(9669):1097-104.

7. Corless CL, Ballman K V., Antonescu CR, et al. Pathologic and molecular features correlate with long-term outcome after adjuvant therapy of resected primary GI stromal tumor: The ACOSOG Z9001 trial. J Clin Oncol. 2014;32(15):1563-70.

8. Joensuu H. One vs three years of adjuvant imatinib for operable gastrointestinal stromal tumor: a randomized trial. JAMA 2012;307(12): 1265 . 
9. Joensuu H. Risk factors for gastrointestinal stromal tumor recurrence in patients treated with adjuvant imatinib. Cancer. 2014;120:2325-33.

10. Joensuu H, Eriksson M, Sundby Hall K, et al. Adjuvant imatinib for high-risk GI stromal tumor: analysis of a randomized trial. $J$ Clin Oncol. 2016;34(3):244-50.

11. Joensuu H, Wardelmann E, Sihto H, et al. Effect of KIT and PDGFRA mutations on survival in patients with gastrointestinal stromal tumors treated with adjuvant imatinib: an exploratory analysis of a randomized clinical trial. JAMA Oncol. 2017;3(5):602-9.

12. Joensuu H, Eriksson M, Hall KS, et al. Three versus one year of adjuvant imatinib for high-risk gastrointestinal stromal tumor (GIST): survival analysis of a randomized trial after 10 years of follow-up. J Clin Oncol. 2020;38 Suppl:abstract no. 11503.

13. Casali PG, Le Cesne A, Velasco AP, et al. Time to definitive failure to the first tyrosine kinase inhibitor in localized GI stromal tumors treated with imatinib as an adjuvant: a European Organisation for Research and Treatment of Cancer Soft Tissue and Bone Sarcoma Group Intergroup Randomized Tr. J Clin Oncol. 2015;33(36):4276-83.

14. Raut CP, Espat NJ, Maki RG, et al. Efficacy and tolerability of 5-year adjuvant imatinib treatment for patients with resected intermediate- or high-risk primary gastrointestinal stromal tumor: the PERSIST-5 clinical trial. JAMA Oncol. 2018;4(12):e184060.

15. Casali PG, Abecassis N, Bauer S, et al. Gastrointestinal stromal tumours: ESMO-EURACAN clinical practice guidelines for diagnosis, treatment and follow-up. Ann Oncol. 2018;29 Suppl 4:iv68-78.

16. NCCN clinical practice guidelines in oncology. Soft tissue sarcoma. Version 6.2019. Available at: https://www.nccn.org/profe ssionals/physician_gls/pdf/sarcoma.pdf.

17. Eisenberg BL, Harris J, Blanke CD, et al. Phase II trial of neoadjuvant/adjuvant imatinib mesylate (IM) for advanced primary and metastatic/recurrent operable gastrointestinal stromal tumor (GIST): early results of RTOG 0132/ACRIN 6665. J Surg Oncol. 2009;99(1):42-7.

18. Wang D, Zhang Q, Blanke CD, et al. Phase II trial of neoadjuvant/adjuvant imatinib mesylate for advanced primary and metastatic/recurrent operable gastrointestinal stromal tumors: long-term follow-up results of Radiation Therapy Oncology Group 0132. Ann Surg Oncol. 2012;19(4):1074-80.

19. McAuliffe JC, Hunt KK, Lazar AJF, et al. A randomized, Phase II study of preoperative plus postoperative imatinib in GIST: evidence of rapid radiographic response and temporal induction of tumor cell apoptosis. Ann Surg Oncol. 2009;16(4):910-19.

20. Rutkowski P, Gronchi A, Hohenberger P, et al. Neoadjuvant imatinib in locally advanced gastrointestinal stromal tumors (GIST): the EORTC STBSG experience. Ann Surg Oncol. 2013;20(9):2937-43.

21. Gold JS, Dematteo RP. Combined surgical and molecular therapy: the gastrointestinal stromal tumor model. Ann Surg. 2006;244(2):176-84.

22. Rutkowski P, Hompes D. Combined therapy of gastrointestinal stromal tumors. Surg Oncol Clin N Am. 2016;25(4):735-59.

23. Keung EZ, Fairweather M, Raut CP. The role of surgery in metastatic gastrointestinal stromal tumors. Curr Treat Options Oncol. 2016;17(2):8.

24. Keung EZ, Raut CP. The management of gastrointestinal stromal tumors. Surg Clin North Am. 2017;97(2):437-52.

25. Raut CP, Posner M, Desai J, et al. Surgical management of advanced gastrointestinal stromal tumors after treatment with targeted systemic therapy using kinase inhibitors. J Clin Oncol. 2006;24(15):2325-31.

26. Bauer S, Rutkowski P, Hohenberger P, et al. Long-term followup of patients with GIST undergoing metastasectomy in the era of imatinib-analysis of prognostic factors (EORTC-STBSG collaborative study). Eur J Surg Oncol. 2014;40(4):412-9.

27. Bischof DA, Kim Y, Blazer DG, et al. Surgical management of advanced gastrointestinal stromal tumors: an international multiinstitutional analysis of 158 patients. $J$ Am Coll Surg. 2014;219(3):439-49.

28. Scaife CL, Hunt KK, Patel SR, et al. Is there a role for surgery in patients with "unresectable" cKIT + gastrointestinal stromal tumors treated with imatinib mesylate? Am $J$ Surg. 2003;186(6):665-9.

29. Sym SJ, Ryu M-H, Lee J-L, et al. Surgical intervention following imatinib treatment in patients with advanced gastrointestinal stromal tumors (GISTs). J Surg Oncol. 2008;98(1):27-33.

30. Zaydfudim V, Okuno SH, Que FG, Nagorney DM, Donohue JH. Role of operative therapy in treatment of metastatic gastrointestinal stromal tumors. J Surg Res. 2012;177(2):248-54.

31. Bonvalot $\mathrm{S}$, Eldweny H, Péchoux $\mathrm{C}$ Le, et al. Impact of surgery on advanced gastrointestinal stromal tumors (GIST) in the imatinib era. Ann Surg Oncol. 2006;13(12):1596-603.

32. Rubió-Casadevall J, Martinez-Trufero J, Garcia-Albeniz X, et al. Role of surgery in patients with recurrent, metastatic, or unresectable locally advanced gastrointestinal stromal tumors sensitive to imatinib: a retrospective analysis of the spanish group for research on sarcoma (GEIS). Ann Surg Oncol. 2015;22(9):2948-57.

33. Fairweather M, Balachandran VP, Li GZ, et al. Cytoreductive surgery for metastatic gastrointestinal stromal tumors treated with tyrosine kinase inhibitors: a 2-institutional analysis. Ann Surg. 2018;268(2):296-302.

34. Du C, Zhou Y, Song C, Wang Y, Jie Z. Is there a role of surgery in patients with recurrent or metastatic gastrointestinal stromal tumours responding to imatinib: a prospective randomised trial in China. Eur J Cancer. 2014;50(10):1772-8.

35. Tielen R, Verhoef C, Coevorden F Van, et al. Surgery after treatment with imatinib and/or sunitinib in patients with metastasized gastrointestinal stromal tumors: is it worthwhile? World J Surg Oncol. 2012;10:111.

36. Raut CP, Wang Q, Manola J, et al. Cytoreductive surgery in patients with metastatic gastrointestinal stromal tumor treated with sunitinib malate. Ann Surg Oncol. 2010;17(2):407-15.

37. Mussi C, Ronellenfitsch U, Jakob J, et al. Post-imatinib surgery in advanced/metastatic GIST: is it worthwhile in all patients? Ann Oncol. 2010;21(2):403-8.

38. DeMatteo RP, Maki RG, Singer S, Gonen M, Brennan MF, Antonescu CR. Results of tyrosine kinase inhibitor therapy followed by surgical resection for metastatic gastrointestinal stromal tumor. Ann Surg. 2007;245(3):347-52.

39. Gronchi A, Fiore M, Miselli F, et al. Surgery of residual disease following molecular-targeted therapy with imatinib mesylate in advanced/metastatic GIST. Ann Surg. 2007;245(3):341-6.

40. Ruka W, Rutkowski P, Szawłowski A, et al. Surgical resection of residual disease in initially inoperable imatinib-resistant/intolerant gastrointestinal stromal tumor treated with sunitinib. Eur $J$ Surg Oncol. 2009;35(1):87-91.

41. Andtbacka RHI, Ng CS, Scaife CL, et al. Surgical resection of gastrointestinal stromal tumors after treatment with imatinib. Ann Surg Oncol. 2007;14(1):14-24.

42. Rutkowski P, Nowecki Z, Nyckowski P, et al. Surgical treatment of patients with initially inoperable and/or metastatic gastrointestinal stromal tumors (GIST) during therapy with imatinib mesylate. J Surg Oncol. 2006;93(4):304-11.

Publisher's Note Springer Nature remains neutral with regard to jurisdictional claims in published maps and institutional affiliations. 